\title{
ESTABLECIMIENTO DEL PASTO ‘CT-115’ (Pennisetum purpureum) EN UNA ZONA SEMIÁRIDA DEL NORESTE DE MEXICO
}

\author{
ESTABLISHMENT OF ‘CT-115’ (Pennisetum purpureum ) GRASS IN A SEMI-ARID \\ AREA OF THE NORTHEAST MÉXICO
}

\author{
José J. Nava Cabelloํㅜ, Erasmo Gutiérrez Ornelas ${ }^{1,3 *}$, Francisco Zavala García ${ }^{1}$, Emilio Olivares Sáenz ${ }^{1}$, José Elías \\ Treviño ${ }^{1}$, Hugo Bernal Barragán ${ }^{1,3}$ y Rafael S. Herrera García ${ }^{2,3}$
}

${ }^{1}$ Facultad de Agronomía, Universidad Autónoma de Nuevo León. Pedro de Alba s/n, Ciudad Universitaria. 66450, San Nicolás de Los Garza, Nuevo León. ${ }^{2}$ Instituto de Ciencia Animal. Apartado Postal 24. San José de Las Lajas. Mayabeque, Cuba. ${ }^{3}$ Red Internacional de Nutrición y Alimentación en Rumiantes.

${ }^{*}$ Autor para correspondencia (egtzo@hotmail.com)

\section{RESUMEN}

El objetivo del experimento fue evaluar el efecto de dos densidades (17 850 y 11350 plantas/ha) y cuatro fechas de siembra (julio, agosto, septiembrey octubre de 2009) en el establecimiento del pasto Pennisetum purpureum cv. 'CT-115'. Se utilizó un diseño completamente al azar con parcelas divididas con dos repeticiones de las parcelas grandes en donde estuvieron asignadas las fechas de siembra; las densidades de siembra fueron asignadas al azar a subparcelas dentro de cada parcela grande. Se muestrearon aleatoriamente siete plantas por unidad experimental para estimar las proporciones de hojas, tallos y material muerto; la relación hoja:tallo; los porcentajes de materia seca (MS) de tallo, número de tallos/planta; y el rendimiento de MS $\left(\mathrm{g} \mathrm{m}^{-2}\right)$. Se registraron datos de temperatura y precipitación. En el mes de mayo del 2010, se evaluó el grado de establecimiento del pasto. Las heladas $\left(-6^{\circ} \mathrm{C}\right)$ ocurridas durante dos días consecutivos en enero de 2010 no afectaron el establecimiento del pasto 'CT-115', ya que rebrotó plenamente en abril ). La densidad de siembra influyó $(P \leq 0.05)$ en la proporción de material muerto el cual fue de $15.8 v s .11 .4 \%$ para las densidades de siembra de 17850 y 11350 plantas/ha, respectivamente, pero no afectó $(\mathrm{P}>0.05)$ al rendimiento de materia seca. Hubo menor porcentaje de MS en tallo en las plantas sembradas en julio y agosto que en las sembradas en fechas posteriores; así mismo, la proporción de hoja fue mayor $(P \leq 0.05)$ en esos primeros dos meses $(57.7$ y 58.5 $\%$; respectivamente). La relación hoja:tallo y el número de tallos/planta fueron también mayores $(P \leq \mathbf{0 . 0 5})$ en las dos primeras fechas de siembra. Se concluye que la producción de materia seca no fue afectada por la densidad y la fecha de siembra, y que las dos heladas de enero 2010 no afectaron el establecimiento del pasto 'CT-115', a pesar de su origen tropical.

Palabras clave: Pennisetum purpureum, densidad de siembra, fecha de siembra, producción de materia seca.

\section{SUMMARY}

The objective of this experiment was to evaluate the effect of two sowing densities (17 850 and 11350 plants/ha) and four planting dates (July, August, September and October 2009) on the establishment of Pennisetum purpureum cv. 'CT-115'. A split-plot design with two repetitions for whole plots was employed and planting dates were randomly assigned. Sowing densities were randomly assigned to the split-plot within each whole plot. Seven plants were randomly sampled in each experimental unit to estimate leaves, stems and senescent material proportions, leaf:stem ratio, dry mater (DM) percentage in stem, number of stems/plant and DM yield $\left(\mathrm{g} \mathrm{m}^{-2}\right)$. Temperature and precipitation were recorded during the two years. Grass establishment was evaluated on May of 2010. The two frosts $\left(-6{ }^{\circ} \mathrm{C}\right)$ recorded during two consecutive days on January of 2010 did not affect 'CT-115' grass establishment since normal re-growth was observed on April. Sowing density affected $(\mathrm{P} \leq 0.05)$ the ratio of senescent material, which was $15.8 v s .11 .4 \%$ for the sowing densities of 17850 and 11350 plants/ha, respectively; it did not however affect $(P>0.05)$ dry matter yield. There was less $(P$ $\leq 0.05) \mathrm{DM}$ in stems of plants sowed on July and August than in further planting dates. Likewise, leaf proportion was higher $(P \leq 0.05)$ in those early months (57.7 and $58.5 \%$; respectively). Leaf:stem ratio and number of stems/plant were higher $(P \leq 0.05)$ in the two first months of planting. It was concluded that dry matter yield was not affected by sowing density or by planting dates, and that the two frosts in January of 2010 did not affect the establishment of 'CT-115' grass, in spite of its tropical origin.

Index words: Pennisetum purpureum, planting density, planting date, dry matter production.

\section{INTRODUCCIÓN}

Los sistemas de producción de ganado del Noreste de México dependen de agostaderos nativos y praderas establecidas principalmente de pasto Buffel común (Cenchrus ciliaris L.; Gómez et al., 2007). En condiciones de sequía y en la época de invierno, la producción de forraje se reduce por lo que los ganaderos alimentan su ganado con pacas de forraje henificado, de la misma especie; en ocasiones emplean pasto Bermuda (Cynodon dactylon var. 'NK-37') y Bermuda Cruza 2 (var. 'Tifton 68'). Además, pueden utilizar grano de sorgo (Sorghum bicolor L. Moench) y sus esquilmos, rastrojos de maíz (Zea mays L.) y en situaciones extremas, nopal (Opuntia sp.). Sin embargo, con frecuencia las pacas o rastrojos presentan bajos contenidos de proteína cruda (menores de $7 \%$ ) y altos valores de fibra detergente neutro (mayores a $75 \%$ ), lo que restringe el consumo de forraje por el ganado (Juárez-Reyes et al., 2009).

En consecuencia, los ganaderos y agricultores han optado por establecer especies alternativas que pudieran representar una mejor opción forrajera, tales como el pasto 'Pretoria 90' (Dichanthium annulatum), Bermuda Cruza 2 y 'Klein' (Panicum coloratum); en estas especies los rendimientos 
dependen de la precipitación anual y fluctúan entre 3 y $8 \mathrm{t}$ MS ha- ${ }^{-1}$ año $^{-1}$ (García et al., 2007) y los contenidos de proteína cruda varían de 6.6 a 10.5 \% (Faria y Sánchez, 2007; Ramírez et al., 2004; Ramírez et al., 2005). Debido a las altas temperaturas y sequías prolongadas que con frecuencia ocurren en la región noreste del país, la mayoría de estos pastos reducen considerablemente su calidad nutricional lo que limita drásticamente la producción animal (Romero et al., 2007)

Existen cultivares del género Pennisetum promisorios para la ganadería, ya que generalmente presentan rendimientos en biomasa superiores a $40 \mathrm{t} \mathrm{ha}^{-1}$ por corte y $120 \mathrm{t}$ ha ${ }^{-1}$ año ${ }^{-1}$ base húmeda (Martínez et al., 1994) y un contenido de proteína cruda de $11.4 \%$ base seca (Valenciaga et al., 2001). En Cuba se desarrolló el clon 'CT-115' (Pennisetum purpureum) resistente a sequía, el cual tiene mayores posibilidades de utilización en el pastoreo directo debido a su baja altura, con rendimientos de biomasa y calidad forrajera aceptables (Martínez et al., 1996).

Con base en la hipótesis de que el cultivar 'CT-115' presenta características de crecimiento y productividad forrajera deseables para las condiciones del noreste de México, el objetivo del presente estudio fue evaluar la productividad de este pasto en dos densidades (17 850 y 11350 plantas/ ha) y cuatro fechas de siembra (julio, agosto, septiembre y octubre de 2009) en la región de Marín, Nuevo León.

\section{MATERIALES Y MÉTODOS}

El experimento se hizo en Marín, N. L., México (25 25' $27^{\prime \prime}$ LN, $100^{\circ} 03^{\prime} 19^{\prime \prime}$ LO, $393 \mathrm{~m}$ de altitud). El clima es extremoso, con precipitación y temperatura promedio anual de $528 \mathrm{~mm}$ y $22^{\circ} \mathrm{C}$, respectivamente $\left(\mathrm{T}_{\text {max }}=42{ }^{\circ} \mathrm{C}\right.$ y $\mathrm{T}_{\text {mín }}$ $=-10^{\circ} \mathrm{C}$ ). Durante el estudio se registraron datos de temperatura máxima, mínima y media, precipitación pluvial y humedad relativa, de los años 2009 y2010, en la estación meteorológica ubicada en el Municipio Marín.

Con el objeto de recabar información de las características de $\mathrm{pH}$, materia orgánica, contenidos de $\mathrm{N}, \mathrm{P}, \mathrm{K}, \mathrm{Ca}$ y $\mathrm{Mg}$ y salinidad del área experimental, se tomaron 10 muestras de suelo $(0 \mathrm{a} 15 \mathrm{~cm})$ antes de la siembra, para formar una muestra compuesta de $1 \mathrm{~kg}$ (Rodríguez, 2002).

\section{Tratamientos y diseño experimental}

La preparación del terreno consistió de un paso de arado y dos pasos de rastra. Los propágulos provinieron de entrenudos de 5 a 6 meses de edad con tres yemas cada uno, edad requerida por este tipo de pastos para lograr un adecuado porcentaje de brotación (Herrera, 2006a). La siembra fue en surcos espaciados a $80 \mathrm{~cm}$ y con distancia entre plantas de 70 y $110 \mathrm{~cm}$, según la densidad de población. Los propágulos se colocaron en el fondo del surco, a una profundidad cercana a $5 \mathrm{~cm}$; posteriormente, se cubrieron con una capa de suelo no mayor a $10 \mathrm{~cm}$.

El riego se aplicó por cintilla con agua de pozo profundo (conductividad eléctrica de $2.9 \mathrm{mS} / \mathrm{cm}$, baja en sodio $\mathrm{y}$ carbonato de sodio). El primer riego fue inmediato a la siembra y después cada $7 \mathrm{~d}$ durante un periodo de $21 \mathrm{~d}$, para garantizar la emergencia; posteriormente, el cultivo solamente recibió el agua de lluvia y no se fertilizó. El periodo de establecimiento se definió de manera que las plantas tuvieran de 5 a 6 meses de edad, y la cosecha se efectuó después del crecimiento de primavera, en mayo de 2010.

Se seleccionaron siete plantas al azar de cada parcela experimental, lo que representó un área de muestreo de 5.6 $\mathrm{m}^{2}$ (17 850 plantas/ha) y $7.7 \mathrm{~m}^{2}$ (11 350 plantas/ha). En estas plantas se midió la altura de la planta; ancho y largo de la cuarta hoja; proporción de hojas, tallos y material muerto; relación hoja:tallo, y número de tallos por planta, y luego se promediaron los valores de las plantas muestreadas para generar el valor correspondiente a cada repetición. Además, en forma manual se separaron hojas, tallos y material muerto para estimar la proporción de hoja:tallo (peso/ peso) y porcentaje del peso de materia seca de cada componente, así como el rendimiento de materia seca $\left(\mathrm{g} \mathrm{m}^{-2}\right)$. Las medidas foliares de largo, ancho y área foliar se hicieron en la cuarta hoja completamente abierta, de acuerdo con la metodología descrita por Herrera (2006b) y por Herrera y Martínez (2006) para detectar diferencias entre clones de Pennisetum.

Los datos de las variables altura de la planta, largo, ancho y área foliar de la cuarta hoja, porcentajes de hojas, tallos y material muerto, relación hoja:tallo, así como número de tallos por planta, porcentajes de materia seca de hojas, tallos y material muerto, además del rendimiento seco (g $\mathrm{m}^{-2}$ ) se analizaron con un un diseño completamente al azar en parcelas divididas, con dos repeticiones de parcelas grandes en donde estuvieron asignadas las fechas de siembra; las densidades de siembra fueron asignadas al azar a subparcelas dentro de cada parcela grande. El área de cada subparcela fue de $225 \mathrm{~m}^{2}$, con un área total del experimento de $3600 \mathrm{~m}^{2}$. Los valores medios, en los casos necesarios, se compararon mediante la prueba de Duncan (Steel y Torrie, 1992). Para el análisis estadístico se aplicó el programa SPSS (2008) versión 17.0.

\section{RESULTADOS Y DISCUSIÓN}

El análisis de suelo ( 0 a $15 \mathrm{~cm}$ de profundidad) del área experimental mostró que es de textura arcillosa, con $\mathrm{pH}$ ligeramente alcalino y contenido medianamente pobre de 
materia orgánica y de nitrógeno (Cuadro 1).

En cuanto a los indicadores del clima durante los 12 meses del periodo experimental, en septiembre de 2009 se registró la mayor precipitación y en enero de 2010 la temperatura promedio mensual más baja (Cuadro 2); en los días 9 y 10 de enero se presentaron temperaturas mínimas de $-6^{\circ} \mathrm{C}$.

Las temperaturas de julio a octubre permitieron el establecimiento del pasto en cada una de las fechas de siembra. A pesar de las bajas temperaturas de enero y febrero no hubo mortalidad de plantas, pues aunque el material vegetativo se heló en la parte aérea, todas las plantas se recuperaron por completo. Como se mencionó, este pasto fue desarrollado en Cuba para su siembra en condiciones tropicales, sin heladas, con temperaturas óptimas para su crecimiento entre 27 y $30{ }^{\circ} \mathrm{C}$ (Herrera, 2006a). Por tanto, los presentes resultados indican que el pasto 'CT-115', además de ser tolerante a la sequía Herrera (2009) también de soporta heladas severas de $-6{ }^{\circ} \mathrm{C}$ durante el establecimiento. Lo anterior pudiera ser relevante en siembras tardías de este pasto, ya que las heladas severas sobre plántulas aún no totalmente establecidas, podrían coadyuvar a disminuir la carga potencial de plagas y competencia con malezas.

No hubo efecto $(\mathrm{P}>0.05)$ de interacción densidad $\mathrm{x}$ fecha de siembra para variable alguna, por lo que a continuación sólo se presentan los resultados de los efectos principales.

Cuadro 1. Propiedades del suelo del área de estudio.

\begin{tabular}{lccc}
\hline Determinación & Cantidad & Interpretación & Método \\
\hline Color & Seco $6 / 2$ & Gris cafesáceo claro & Escala Munsell \\
$\mathrm{pH}$ & 7.8 & Ligeramente alcalino & Potenciómetro \\
Textura & Arcilla $61.4 \%$ & Arcilloso & Hidrómetro de Bouyoucos \\
Materia orgánica & $1.71 \%$ & Medianamente pobre & Walkley-black \\
Nitrógeno & $0.134 \%$ & Medianamente pobre & Kjeldahl \\
Fósforo & $64.3 \mathrm{ppm}$ & Óptimo & Olsen modificado \\
Potasio & $1.42 \mathrm{meq} / 100 \mathrm{gr}$ & Óptimo & Absorción atómica \\
Sales solubles totales & $1.99 \mathrm{mS} / \mathrm{cm}$ & No salino & Extracto de saturación \\
\hline
\end{tabular}

Cuadro 2. Promedios mensuales de precipitación pluvial $(\mathrm{PP}, \mathrm{mm})$, temperaturas máxima, mínima y media $\left({ }^{\circ} \mathrm{C}\right)$, y humedad relativa (\%), en Marín, Nuevo León, México, de julio 2009 a junio 2010.

\begin{tabular}{lrcccc}
\hline \multirow{2}{*}{ Fecha } & PP & \multicolumn{4}{c}{ Temperatura } \\
\cline { 3 - 5 } & & Máxima & Mínima & Media & Humedad relativa \\
\hline Julio 2009 & 4.4 & $39.5 \mathrm{e}$ & 24.4 & 31.2 & 51.2 \\
Agosto & 70.0 & 39.0 & 23.5 & 30.7 & 50.4 \\
Septiembre & 138.0 & 31.7 & 21.0 & 25.4 & 76.4 \\
Octubre & 45.6 & 28.7 & 17.9 & 22.7 & 74.7 \\
Noviembre & 21.6 & 26.0 & 10.1 & 17.8 & 64.1 \\
Diciembre & 30.2 & 19.7 & 7.3 & 12.7 & 78.2 \\
Enero 2010 & 40.0 & 20.0 & 6.5 & 12.9 & 69.0 \\
Febrero & 21.0 & 20.8 & 6.8 & 13.4 & 67.3 \\
Marzo & 14.2 & 27.4 & 9.6 & 18.8 & 55.2 \\
Abril & 106.4 & 29.9 & 17.2 & 23.2 & 60.1 \\
Mayo & 20.8 & 34.2 & 20.6 & 27.0 & 50.2 \\
Junio & 89.2 & 37.3 & 23.6 & 29.6 & 55.3 \\
\hline
\end{tabular}




\section{Efecto de la densidad de siembra}

Con excepción del material muerto (Cuadro 3), en ninguna variable considerada en este estudio se detectaron diferencias entre las dos densidades de siembra, aunque las praderas sembradas con 17850 plantas/ha produjeron $38 \%$ más de material muerto. El rendimiento de materia seca fue $19 \%$ mayor en alta densidad que en las parcelas sembradas con 11350 plantas/ha (Cuadro 4), pero la diferencia no fue significativa, tal vez debido al bajo número de repeticiones utilizadas para este factor.

Los rendimientos de materia seca aquí obtenidos fueron $20 \%$ inferiores a los de Padilla y Curbelo (2005) para este mismo pasto en Cuba, lo cual se atribuye al suelo arcilloso y ligeramente alcalino de Marín, N.L. (Cuadro 1) y a las bajas precipitaciones asociadas con altas temperaturas de su clima de trópico seco (Cuadro 2), mientras que en Cuba el suelo es de alta fertilidad, y el sitio recibe más lluvia y su temperatura es menor, características propias del trópico húmedo.

La ausencia de diferencias en el contenido (\%) de materia seca en hojas, tallos y material muerto (Cuadro 4) se puede atribuir a que el número de plantas existentes en cada densidad de siembra no afectó el contenido de agua de los órganos de la planta. Herrera y Ramos (2006) informaron similares resultados al evaluar diferentes densidades de siembra en Pennisetum purpureum cv. 'King grass' y $P$. purpureum cv. 'CT-115'.

\section{Efecto de las fechas de siembra}

El mayor número de tallos/planta $(\mathrm{P} \leq 0.05)$ se obtuvo en las primeras dos fechas de siembra (Cuadro 5), lo que refleja el efecto de la edad de este grupo de plantas, tal vez debido a que la helada obligó a remover todo el material muerto de las plantas de esas fechas. Estas parcelas al mes de diciembre mostraron las características propias de una pradera ya establecida (altura, hojas inferiores senescentes, entre otros indicadores), mientras que el pasto sembrado en las parcelas de septiembre y octubre apenas mostró en diciembre un crecimiento incipiente de hojas y tallos, con una altura menor a $30 \mathrm{~cm}$.

El rebrote anual del pasto inició con la precipitación de $106 \mathrm{~mm}$ durante el mes de abril. Aunque en las fechas de siembra de julio y agosto se encontró mayor número de tallos/planta $(\mathrm{P} \leq 0.05)$, esto no se reflejó en diferencias en el rendimiento de materia seca (Cuadro 5). En la región de Marín, N. L. la precipitación que se recibe en primavera normalmente no es suficiente para promover el máximo crecimiento de las plantas; el pasto tuvo un periodo de crecimiento de $45 \mathrm{~d}$ y alcanzó un rendimiento $50 \%$ menor al reportado en Cuba por Fundora et al. (2005).

Las proporciones de hoja y tallo, así como la relación hoja:tallo presentaron diferencias $(\mathrm{P} \leq 0.05)$ entre fechas, a favor de las siembras de julio y agosto, quizás debido a que en estas fechas hubo un mayor tiempo de establecimiento y más oportunidad de acumular horas calor, lo que permitió

Cuadro 3. Promedios de altura de planta, ancho, largo y área foliar de la $4^{\mathrm{a}}$ hoja; porcentajes de hoja, tallo y material muerto (MM); relación hoja:tallo y número de tallos/planta en Pennisetum purpureum var. 'CT-115' sembrado en dos densidades de plantas, en Marín, N. L.

\begin{tabular}{lccccccccc}
\hline $\begin{array}{l}\text { Densidad } \\
\text { (plantas/ha) }\end{array}$ & $\begin{array}{c}\text { Altura } \\
(\mathrm{cm})\end{array}$ & $\begin{array}{c}\text { Ancho } \\
(\mathrm{cm})\end{array}$ & $\begin{array}{c}\text { Largo } \\
(\mathrm{cm})\end{array}$ & $\begin{array}{c}\text { Área } \\
\left(\mathrm{cm}^{2}\right)\end{array}$ & Hoja (\%) & Tallo (\%) & MM (\%) & $\begin{array}{c}\text { Relación } \\
\text { Hoja/Tallo }\end{array}$ & $\begin{array}{c}\text { Núm. de } \\
\text { tallos/planta }\end{array}$ \\
\hline 17850 & 40.3 & 3.7 & 98.0 & 360.5 & 49.1 & 35.1 & $15.8 \mathrm{a}$ & 1.5 & 22.8 \\
11350 & 38.4 & 3.6 & 99.8 & 360.0 & 53.1 & 35.4 & $11.4 \mathrm{~b}$ & 1.6 & 25.3 \\
ee \pm & 3.84 & 0.17 & 2.09 & 23.57 & 2.81 & 2.0 & 1.82 & 0.17 & 2.18 \\
\hline
\end{tabular}

Medias con letras iguales en columna MM, no son estadísticamente diferentes (Duncan, 0.05); ee = error estándar; MM = material muerto.

Cuadro 4. Rendimiento de materia seca, porcentajes de materia seca de hoja, tallo, material muerto y planta completa, en Pennisetum purpureum var. 'CT-115' sembrado en dos densidades de siembra, en Marín, N. L.

\begin{tabular}{lccccc}
\hline \multirow{2}{*}{ Densidad plantas/ha } & \multicolumn{2}{c}{ Rendimiento $\left(\mathrm{g} \mathrm{m}^{-2}\right)$} & \multicolumn{3}{c}{ Contenido (\%) de materia seca en } \\
\cline { 3 - 6 } & & Hoja & Tallo & Material muerto & Planta completa \\
\hline 17850 & 560 & 28.9 & 19.6 & 73.1 & 27.1 \\
11350 & 470 & 29.6 & 18.4 & 64.9 & 25.8 \\
ee \pm & 0.95 & 0.61 & 0.64 & 5.0 & 1.11 \\
\hline
\end{tabular}

ee = error estándar. 
una mayor producción de hojas. El porcentaje de hojas fue $45 \%$ mayor en las siembras de julio y agosto que lo reportado en Cuba por Herrera (2009).

La proporción de tallo fue mayor en $45 \%(\mathrm{P} \leq 0.05)$ en el pasto sembrado durante septiembre y octubre, respecto a julio y agosto (Cuadro 6); en cambio, la relación hoja:tallo fue mayor $(\mathrm{P} \leq 0.05)$ en julio y agosto $(2.0$ y 2.1$)$ que en las siembras de septiembre y octubre $(1.0$ y 1.1$)$. En las siembras de julio y agosto, el contenido de materia seca del tallo fue $21.2 \%$ menor que en las de septiembre y octubre.

\section{CONCLUSIONES}

El pasto Pennisetum purpureum cv. 'CT-115' se estableció adecuadamente en Marín, N. L., región representativa del trópico seco mexicano, donde se estudia por primera vez. La producción de materia seca no fue afectada por la densidad ni la fecha de siembra. Las dos heladas de $-6{ }^{\circ} \mathrm{C}$ ocurridas durante el mes de enero de 2010 no influyeron en el establecimiento de este pasto. En condiciones similares a las presentadas durante el experimento, el pasto 'CT-115' puede ser sembrado de julio a octubre en la región Noreste de México, sin afectar su establecimiento.

\section{AGRADECIMIENTOS}

Al Consejo Nacional de Ciencia y Tecnología (CONACYT), Fundación Produce Nuevo León A. C. y la Secretaría de Educación Pública (SEP), por el apoyo brindado para la realización de la presente investigación.

\section{BIBLIOGRAFÍA}

De la Fuente, H (2010) Caracterización del clima en Nuevo León. Informe de actividades INIFAP-Fundación Produce Nuevo León, A. C. http://clima.inifap.gob.mx (Agosto, 2010).

Faria M J, A Sánchez (2007) Efecto del aplazamiento de utilización sobre el contenido de nutrientes y digestibilidad de la materia orgánica de la asociación Buffel-Leucaena. Interciencia 32:185-187.

Fundora O, A Otero, M E González, F Sierra (2005) Uso del Pennisetun purpureum (Clon Cuba CT-115) como banco de biomasa para búfalas de río y su efecto en el control de malezas. Rev. Cub. Ciencia Agríc. 39:569-573.

García D J G, R G Ramírez Lozano, R Morales Rodríguez G García Díaz (2007) Ruminal digestion and chemical composition of new genotypes of Buffelgrass (Cenchrus ciliaris) under irrigation and fertilization. Interciencia 32:349-353

Gómez de la F E, H Díaz Solís, A Saldivar Fitzmaurice, F Briones Encinia, V Vargas Tristán, W E Grant (2007) Patrón de crecimiento [Pennisetum ciliare L. (Link.) Sin. Cenchrus ciliaris L.] en Tamaulipas, México. Téc. Pec. Méx. 45:1-17

Herrera R S (2006a) Siembra y establecimiento de pastizales de gramíneas. In: Manual de Pastos y Forrajes. EDICA, La Habana, Cuba. (CDROM).

Herrera R S (2006b) Fisiología, calidad y muestreos. In: Fisiología, Producción de Biomasa y Sistemas Silvopastoriles en Pastos Tropicales. Abono Orgánico y Biogás. R S Herrera, I Rodríguez, G Febles

Cuadro 5. Rendimiento de materia seca, altura y número de tallos por planta a los $45 \mathrm{~d}$ de rebrote, en Pennisetum purpureum var. 'CT-115' sembrado en cuatro fechas de siembra, en Marín, N. L.

\begin{tabular}{lccc}
\hline Fecha de siembra & Rendimiento de materia seca $\left(\mathrm{g} \mathrm{m}^{-2}\right)$ & Altura $(\mathrm{cm})$ & Tallos/planta \\
\hline Julio & 540 & 33.1 & $35.3 \mathrm{a}$ \\
Agosto & 600 & 36.9 & $29.2 \mathrm{a}$ \\
Septiembre & 480 & 44.3 & $16.0 \mathrm{~b}$ \\
Octubre & 420 & 43.2 & $15.8 \mathrm{~b}$ \\
ee \pm & 1.3 & 5.4 & 3.1 \\
\hline
\end{tabular}

Medias con letras iguales en la variable tallos/planta, no son estadísticamente diferentes (Duncan, 0.05); ee = error estándar.

Cuadro 6. Promedios de proporciones de hoja, tallo y, material muerto (MM); relación oja:tallo ( $\mathrm{R} H / \mathrm{T})$ y porcentajes de materia seca en hoja, tallo, material muerto y planta completa, en Pennisetum purpureum var. 'CT-115' sembrado en cuatro fechas de siembra, en Marín, N. L.

\begin{tabular}{|c|c|c|c|c|c|c|c|c|}
\hline \multirow{2}{*}{ Fecha } & \multicolumn{3}{|c|}{$\%$} & \multirow{2}{*}{$\begin{array}{l}\text { Rel. } \\
\text { H/T }\end{array}$} & \multicolumn{4}{|c|}{ Contenido de materia seca (\%) } \\
\hline & Hoja & Tallo & $\mathrm{MM}$ & & Hoja & Tallo & $\mathrm{MM}$ & Planta completa \\
\hline Julio & $57.7 \mathrm{a}$ & $28.0 \mathrm{~b}$ & 14.3 & $2.0 \mathrm{a}$ & 28.0 & $16.6 \mathrm{~b}$ & 66.8 & 25.3 \\
\hline Agosto & $58.5 \mathrm{a}$ & $29.4 \mathrm{~b}$ & 12.1 & $2.1 \mathrm{a}$ & 30.1 & $16.8 \mathrm{~b}$ & 56.9 & 25.7 \\
\hline Septiembre & $44.6 \mathrm{~b}$ & $43.9 \mathrm{a}$ & 11.5 & $1.0 \mathrm{~b}$ & 30.2 & $21.6 \mathrm{a}$ & 76.4 & 27.4 \\
\hline Octubre & $43.7 \mathrm{~b}$ & $39.8 \mathrm{a}$ & 16.4 & $1.1 \mathrm{~b}$ & 28.8 & $20.8 \mathrm{a}$ & 76.1 & 27.4 \\
\hline ee \pm & 4.0 & 2.8 & 2.6 & 0.24 & 1.8 & 0.9 & 7.1 & 1.6 \\
\hline
\end{tabular}

Medias con letras iguales en cada columna, no son estadísticamente diferentes (Duncan, 0.05); ee = error estándar; MM = material muerto. 
(eds). EDICA, La Habana, Cuba. pp:1-108.

Herrera R S, R O Martínez (2006) Mejoramiento genético por vías no clásicas. In: Pennisetum purpureum Para la Ganadería Tropical. R S Herrera, G Crespo, G Febles (eds). EDICA, La Habana, Cuba. pp:15-38.

Herrera R S, N Ramos (2006) Evaluación agronómica y calidad. In: Pennisetum purpureum Para la Ganadería Tropical. R S Herrera, G Crespo, G Febles (eds.). EDICA, La Habana, Cuba. pp:101-124.

Herrera R S (2009) Mejoramiento de Pennisetum purpureum en Cuba. Rev. Cub. Ciencia Agríc. 43 :345-349.

Juárez-Reyes A S, M A Cerrillo-Soto, E Gutiérrez Ornelas, E M Romero-Treviño, J Colín-Negrete, H Bernal Barragán (2009) Estimación del valor nutricional de pastos tropicales a partir de análisis convencionales y de la producción de gas in vitro. Téc. Pec. Méx. 47:55-67.

Martínez R O, R S Herrera, R Cruz, R Tuero, M García (1994) Producción de biomasa con hierba elefante (Pennisetum purpureum) y caña de azúcar (Saccharum officinarum) para la producción animal en el trópico. I. Rendimientos. Rev. Cub. Ciencia Agríc. 28:221-225.

Martínez R O, R S Herrera, V Torres (1996) Cultivo de tejido y fitotecnia de las mutaciones en pastos tropicales Pennisetum purpureum. Otro ejemplo para la obtención de nuevos clones. Rev. Cub. Ciencia Agríc. 30:1-11.
Padilla C, F Curbelo (2005) Dos métodos de plantación en el establecimiento de yerba elefante CT-115 (Pennisetum purpureum). Rev. Cub. Ciencia Agrí. 39:219-222.

Ramírez L G, F W Haenlein, C G García-Castillo, M A Núñez-González (2004) Protein, lignin and mineral contents and in situ dry matter digestibility of native Mexican grasses consumed by range goats. Small Rum. Res. 52:261-269.

Ramírez R G, H González-Rodríguez, G García-Dessommes, R Morales-Rodríguez (2005) Seasonal trends in the chemical composition and digestion of Dichanthium annulatum (Forssk.) Stapf. J. App. Animal Res. 28:35-40.

Rodríguez H (2002) Métodos de Análisis de Suelo y Plantas. Ed. Trillas. México. D.F. pp:9-20.

Romero E M, E Gutiérrez, H Bernal, H Morales, J Colín, E Olivares, O Gutiérrez, V Torres, H Dennis (2007) Seasonability in the concentration of blood metabolites of Charolais and Beefmaster cows grazing Buffel grass in Northeastern Mexico. Cub. J. Agric. Sci. 41:225-230.

SPSS (2008) Statistical Package for Social Sciences. User's Manual (Release 17.0) http://www.spss.com

Steel R G D, J H Torrie (1992) Bioestadistica: Principios y Procedimientos. 2da. ed. McGraw Hill/Interamericana de México. 622 p.

Valenciaga D, B Chongo, O La O (2001) Characterization of Pennisetum CUBA CT 115 clone. Chemical composition and rumen DM degradability. Cub. J. Agric. Sci. 35:325-329. 